\title{
Near-Capacity Irregular Bit-Interleaved Coded Modulation
}

\author{
R. Y. S. Tee, R. G. Maunder, J. Wang and L. Hanzo \\ School of ECS, University of Southampton, SO17 1BJ, UK. \\ http://www-mobile.ecs.soton.ac.uk
}

\begin{abstract}
An Irregular Bit-Interleaved Coded Modulation based Iterative Decoding (Ir-BICM-ID) aided scheme is proposed. The irregularity of the scheme pervades the three basic components of BICM-ID, namely the encoder, the unity-rate precoder and the bit-to-symbol mapper. As a result, adaptive BICM-ID schemes constituted by irregular components are created, which are capable of approaching the capacity of coded modulation. This is achieved by creating a narrow EXtrinsic Information Transfer (EXIT) chart, using a novel EXIT curve matching algorithm. The proposed Ir-BICM-ID scheme employs Irregular Convolutional Codes (IrCC), Irregular Unity-Rate Codes (IrURC) and Irregular Mappers (IrMapper).
\end{abstract}

\section{INTRODUCTION}

Bit-Interleaved Coded Modulation using Iterative Decoding (BICM-ID) [1] [2] was originally designed as a bandwidth efficient coded modulation scheme for Rayleigh fading channels. It consists of three main blocks - a convolutional encoder, bit-interleavers and a bit-to-symbol mapper. Iterative detection was achieved by exchanging soft extrinsic information between the symbol-to-bit demapper and the decoder. This BICM-ID scheme exhibits a turbo-like cliff and the achievable convergence performance can be characterized with the aid of EXtrinsic Information Transfer (EXIT) charts [3].

Different bit-to-symbol mapping schemes have been investigated in order to improve the convergence behaviour of BICM-ID [4] [5] by shaping the demapper's EXIT characteristic for the sake of creating an open EXIT tunnel and hence to achieve an infinitesimally low Bit Error Ratio (BER). Furthermore, an adaptive BICM arrangement using various iterative decoding schemes was proposed in [6], which employed different signal constellations and bit-to-symbol mapping within one codeword. This flexible signalling scheme required the knowledge of the average signal quality at the transmitter, for invoking advanced Adaptive Modulation and Coding (AMC) [7] in order to improve the overall BICM-ID scheme's achievable performance.

A Unity-Rate Code (URC) can be used as a precoder for creating an Infinite Impulse Response (IIR) inner demapper component in order to reach the $(1,1)$ EXIT chart convergence point [8] and hence to achieve an infinitesimally low BER. A precoded mapper scheme was also proposed for a three-stage - encoder, precoder and modulator - design constituted by the bit-based or symbol-based precoder [9]. Furthermore, a flexible irregular demapper combined with modulation doping was proposed in [10] for the sake of producing an open EXIT tunnel.

Motivated by the aforementioned adaptive BICM-ID schemes, in this paper, we propose an irregular BICM-ID arrangement for the sake of achieving a near-capacity performance. Our approach is based on invoking EXIT chart analysis for minimising the area of the open EXIT tunnel in order to achieve a near-capacity performance. The classic outer convolutional encoder is replaced by an Irregular Convolutional Code (IrCC) [11] [12] which has a range of different coding rates based on a punctured convolutional mother code. In the same spirit, the inner component consists of an Irregular Unity Rate

The financial support of the EPSRC, UK and that of the European Union is gratefully acknowledged.
Code (IrURC) combined with an Irregular Mapper (IrMapper). The inner iterations are invoked between the IrURC having a code rate of unity as well as up to three shift-register stages and the IrMapper employing different mapping schemes for the sake of creating a diverse range of EXIT curves. A high-flexibility inner-outer EXIT chart matching algorithm is used for creating a narrow EXIT tunnel.

The rest of this contribution is organized as follows. Section II provides an overview of our system, outlining the proposed Ir-BICMID scheme. Our EXIT chart analysis is presented in Section III and the irregular components of the Ir-BICM-ID scheme are discussed in Section IV. The EXIT matching algorithm advocated is described in Section V. In Section VI, we quantify the achievable performance of this novel scheme, invoking the IrCC, IrURC and IrMapper, while our conclusions are presented in Section VII.

\section{System OVERVIEW}

The schematic of the proposed Ir-BICM-ID scheme is shown in Figure 1 . The binary source bit stream $u_{1}$ is encoded by the $\mathrm{IrCC}$ encoder and the encoded bits $v_{1}$ are interleaved by the bit interleaver $\pi_{1}$, yielding the permuted bits $u_{2}$. The bit stream $u_{2}$ is then fed into the IrURC encoder and the resultant encoded bits $v_{2}$ are interleaved by the bit interleaver $\pi_{2}$. As seen in Figure 1, the permuted bits $u_{3}$ are then mapped to the input of an irregular modulation scheme, invoking an adaptive mapping scheme referred to as the IrMapper. The modulated symbols $x$ are transmitted via a Rayleigh fading channel and the received signals $y$ are demodulated.

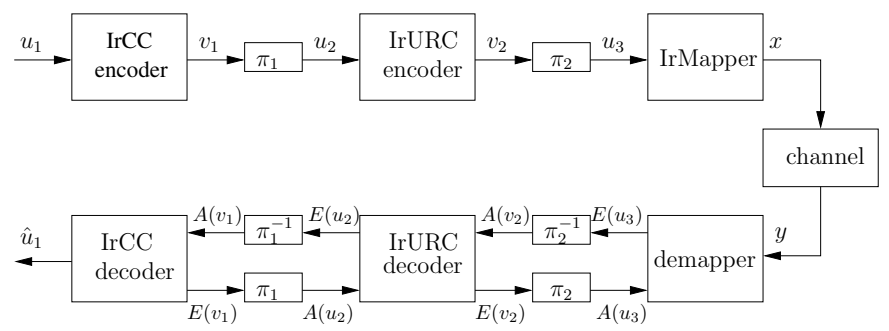

Fig. 1. The Ir-BICM-ID scheme.

At the receiver, an iterative decoder is invoked, exchanging extrinsic information between the inner and outer components. The inner iterations exchange extrinsic information between the irregular demapper and the IrURC decoder, where the notation $A($.$) represents$ the $a$ priori information quantified in terms of Log-Likelihood Ratios (LLRs), while $E($.$) denotes the extrinsic information also expressed$ in terms of LLRs.

By contrast, the outer iterations are invoked between the IrURC decoder and the IrCC decoder of Figure 1. Since both the IrURC and IrCC schemes are trellis-based, their decoders employ the Maximum A-Posteriori (MAP) algorithm. The order of iterative decoding is as follows:

- the inner iterative decoder continues iterations, until no more mutual information improvement is achieved.

- this is then followed by outer iterative decoding. 


\section{EXIT CHART ANALYSIS}

EXIT chart analysis is performed to characterise the achievable iterative decoding convergence performance. The Ir-BICM-ID scheme is a three-stage arrangement, which requires 3-Dimensional (3D) EXIT analysis, as presented in [13]. However, since the IrURC decoder and the Irregular Demapper (Ir-Demapper) constitute lowcomplexity components, the low-complexity inner iterations are continued until the mutual information $E\left(v_{2}\right)$ becomes constant, because no more extrinsic information may be gleaned by any of these two component, without the third component's intervention. Hence, we can simplify the three-stage EXIT chart representation to a 2Dimensional (2D) EXIT curve, as detailed below.

Let $I_{A(b)}$ represent the mutual information between the a prior $i$ information $A(b)$ and the bit $b$, while $I_{E(b)}$ denotes the mutual information between the extrinsic information $E(b)$ and the bit $b$. Observe from Figure 1 that the EXIT function of the Ir-Demapper can be represented as a function of $I_{E\left(u_{3}\right)}$ and the channel's $E_{b} / N_{0}$ value as follows:

$$
I_{E\left(u_{3}\right)}=f_{u_{3}}\left[I_{A\left(u_{3}\right)}, E_{b} / N_{0}\right] .
$$

Observe in Figure 1 that in a typical three-stage concatenated design, the IrURC decoder has two mutual information outputs, namely $I_{E\left(u_{2}\right)}$ and $I_{E\left(v_{2}\right)}$. Both of these mutual information components depend on two a priori mutual information inputs, namely on $I_{A\left(u_{2}\right)}$ and $I_{A\left(v_{2}\right)}$. The two functions can be defined as follows:

$$
\begin{aligned}
& I_{E\left(u_{2}\right)}=f_{u_{2}}\left[I_{A\left(u_{2}\right)}, I_{A\left(v_{2}\right)}\right], \\
& I_{E\left(v_{2}\right)}=f_{v_{2}}\left[I_{A\left(u_{2}\right)}, I_{A\left(v_{2}\right)}\right] .
\end{aligned}
$$

In this Ir-BICM-ID scheme, we continue invoking inner iterations, until we succeed in generating a reliable mutual information $I_{E\left(u_{2}\right)}$, before activating the outer iterations. Hence we may combine the inner component blocks according to Figure 2. The EXIT function of the inner component can be defined by

$$
I_{E\left(u_{2}\right)}=f_{u_{2}}\left[I_{A\left(u_{2}\right)}, E_{b} / N_{0}\right]
$$

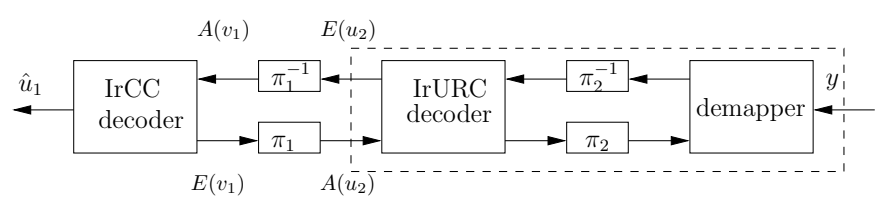

Fig. 2. The Ir-BICM-ID inner block.

For the IrCC decoder, the EXIT function becomes

$$
I_{E\left(v_{1}\right)}=f_{v_{1}}\left[I_{A\left(v_{1}\right)}\right] .
$$

The so-called area-property of EXIT charts can be exploited for creating a near-capacity Ir-BICM-ID scheme based on EXIT curve matching. The area property of EXIT charts [14] states that the area under the EXIT curve of an inner decoder component is approximately equal to the attainable channel capacity, provided that the channel's input is uniformly distributed. As for the outer component, the area under its EXIT function is equivalent to $\left(1-R_{o}\right)$, where $R_{o}$ is the outer component's coding rate. The area properties were shown to hold for the Binary Erasure Channel (BEC), but there is experimental evidence that it also holds for AWGN [13] and Rayleigh fading channels [15].

Let $A_{v_{1}}$ and $\bar{A}_{v_{1}}$ be the areas under the EXIT function of $f_{v_{1}}(i)$ and $f_{v_{1}}^{-1}(i)$, where $i \in[0,1]$ which can be defined as

$$
A_{v_{1}}=\int_{0}^{1} f_{v_{1}}(i) d i \quad \bar{A}_{v_{1}}=\int_{0}^{1} f_{v_{1}}^{-1}(i) d i=1-A_{v_{1}} .
$$

Therefore the area $\bar{A}_{v_{1}}$ under the inverse of the EXIT function is approximately equivalent to the coding rate, $\bar{A}_{v_{1}} \approx R_{o}$. Since the IrURC has a coding rate of unity, the area $A_{v_{2}}$ under the combined inner component block's EXIT curve in Figure 2, can be defined as follows:

$$
A_{v_{2}} \approx C_{\text {channel }}
$$

where $C_{\text {channel }}$ is the uniform-input channel capacity. Our aim is to create a near-capacity design associated with a narrow EXIT tunnel between the inner and outer EXIT function.

\section{IRREGULAR COMPONENTS}

\section{A. Irregular Outer Component}

We employ an outer IrCC component proposed by Tüchler [12], which consists of different-rate components created from a mother code by puncturing. To be more specific, the IrCC was designed from a $1 / 2$-rate memory- 4 mother code defined by the generator polynomial $\left(G_{1}, G_{2}\right)=(31,27)_{8}$ in octal form, where puncturing was employed to generate a set of codes having gradually increasing coding rates. For lower code rates, two additional generator polynomials, namely $G_{3}=(35)_{8}$ and $G_{4}=(33)_{8}$ are employed. The total number of subcodes in the IrCC arrangement used is 17 , having the code rate spanning the range of [0.1,0.9], with a step size of 0.05 .

Each of these 17 subcodes encodes a specific fraction of the bit stream $u_{1}$ of Figure 1 according to a specific weighting coefficient $\alpha_{i}, i=1,2, \ldots, 17$. More specifically, let us assume that there are $L$ number of encoded bits $v_{1}$ in Figure 1, where each subcode $i$ encodes a fraction of $\alpha_{i} r_{i} L$ and generates $\alpha_{i} L$ encoded bits using a coding rate of $r_{i}$. Let us assume that there are $P$ number of subcodes and that the following conditions must be satisfied:

$$
\begin{aligned}
\sum_{i=1}^{P} \alpha_{i} & =1 \\
R_{o} & =\sum_{i=1}^{P} \alpha_{i} r_{i}
\end{aligned}
$$

where $\alpha_{i} \in[0,1], \forall i$ and $R_{o}$ is the average outer code rate. The EXIT functions of all $P=17$ IrCC subcodes are shown in Figure 3.

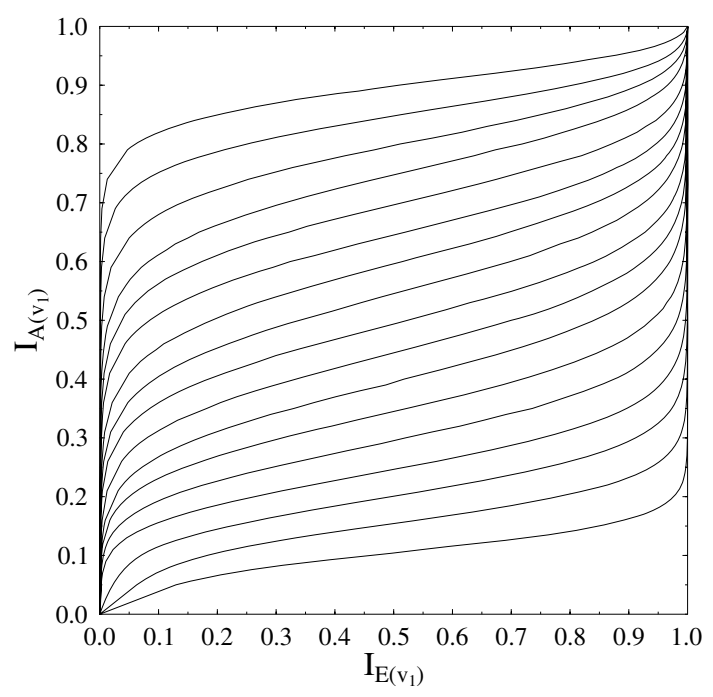

Fig. 3. EXIT functions of the 17 subcodes of the IrCC scheme. 


\section{B. Irregular Inner Component}

In order to generate a narrow open EXIT chart tunnel but nonetheless, which leads to the convergence point of $\left(I_{A}, I_{E}\right)=(1,1)$, we have to design inner EXIT functions which match the shape of those in Figure 3 and exhibit a variety of EXIT characteristic shapes. Again, in this paper, we propose an inner decoder block, which consists of an IrURC and IrMapper combination.

The IrURC scheme consists of three URCs, each having a different memory length. We evaluated the EXIT chart of all possible combinations of up to three different-memory URCs and then selected the three most dissimilar URC EXIT functions, having a generator polynomial $\left(G_{1}, G_{2}\right)$ of $(2,3)_{8},(4,7)_{8}$ and $(16,17)_{8}$. We term them as $\mathrm{URC}_{1} \mathrm{URC}_{2} \mathrm{URC}_{3}$, respectively.

Finally, the IrMapper of Figure 1 consists of irregular mapping schemes, each invoking a different bit-to-symbol mapping strategy. Here, we consider an 8-PSK constellation and employ four different mapping schemes, which exhibit dissimilar EXIT functions, namely Gray Mapping (GM), Ungerböck's Partitioning (UP) [16], Block Partitioning (BP) and Mixed Partitioning (MP) [17]. The corresponding bit-to-symbol mapping schemes are detailed in Table I.

\begin{tabular}{|c||c|}
\hline Mapping Type & $\begin{array}{c}\text { Mapping Indices to Corresponding } \\
\text { Signal Points }(\cos 2 \pi i / M, \sin 2 \pi i / M) \text { for } \\
i \in 012\end{array}$ \\
\hline \hline GM & 013267567 \\
UP & 01234567 \\
BP & $\begin{array}{l}0 \\
\text { MP }\end{array}$ \\
\hline
\end{tabular}

TABLE I

DiFFERENT BIT-TO-SYMBOL MAPPING STRATEGIES : GRAY MAPPING (GM), UNGERBÖCK's PARTITIONING (UP), BLOCK PARTITIONING (BP) AND MiXed PARTITIONING (MP) [17], WHERE $M$ IS THE NUMBER OF CONSTELLATION POINTS.

With the IrURC and IrMapper schemes defined, we proceed by creating 12 different EXIT functions for the inner decoder components, each invoking a different combination of the IrURC and IrMapper schemes. For example, the $\mathrm{URC}_{1}$ scheme employing the GM arrangement was defined in Table II as $\mathrm{UM}_{1}$.

\begin{tabular}{|c||c|c|}
\hline Inner Component & URC Type & Mapping Type \\
\hline \hline $\mathrm{UM}_{1}$ & $\mathrm{URC}_{1}$ & $\mathrm{GM}$ \\
$\mathrm{UM}_{2}$ & $\mathrm{URC}_{2}$ & $\mathrm{GM}$ \\
$\mathrm{UM}_{3}$ & $\mathrm{URC}_{3}$ & $\mathrm{GM}$ \\
$\mathrm{UM}_{4}$ & $\mathrm{URC}_{1}$ & $\mathrm{UP}$ \\
$\mathrm{UM}_{5}$ & $\mathrm{URC}_{2}$ & $\mathrm{UP}$ \\
$\mathrm{UM}_{6}$ & $\mathrm{URC}_{3}$ & $\mathrm{UP}$ \\
$\mathrm{UM}_{7}$ & $\mathrm{URC}_{1}$ & $\mathrm{BP}$ \\
$\mathrm{UM}_{8}$ & $\mathrm{URC}_{2}$ & $\mathrm{BP}$ \\
$\mathrm{UM}_{9}$ & $\mathrm{URC}_{3}$ & $\mathrm{BP}$ \\
$\mathrm{UM}_{10}$ & $\mathrm{URC}_{1}$ & $\mathrm{MP}$ \\
$\mathrm{UM}_{11}$ & $\mathrm{URC}_{2}$ & $\mathrm{MP}$ \\
$\mathrm{UM}_{12}$ & $\mathrm{URC}_{3}$ & $\mathrm{MP}$ \\
\hline
\end{tabular}

TABLE II

VARIOUS URC MAPPER (UM) COMBINATIONS, EACH EXHIBITING A DIFFERENT INNER EXIT FUNCTION.

The EXIT functions of the $Q=12$ combined inner IrURCIrMapper components are plotted in Figure 4 for $E_{b} / N_{0}=5.3 \mathrm{~dB}$. The weighting coefficients are defined as $\beta$, satisfying the following conditions:

$$
\sum_{i=1}^{P} \beta_{i}=1 \quad \text { and } \beta_{i} \in[0,1], \quad \forall i .
$$

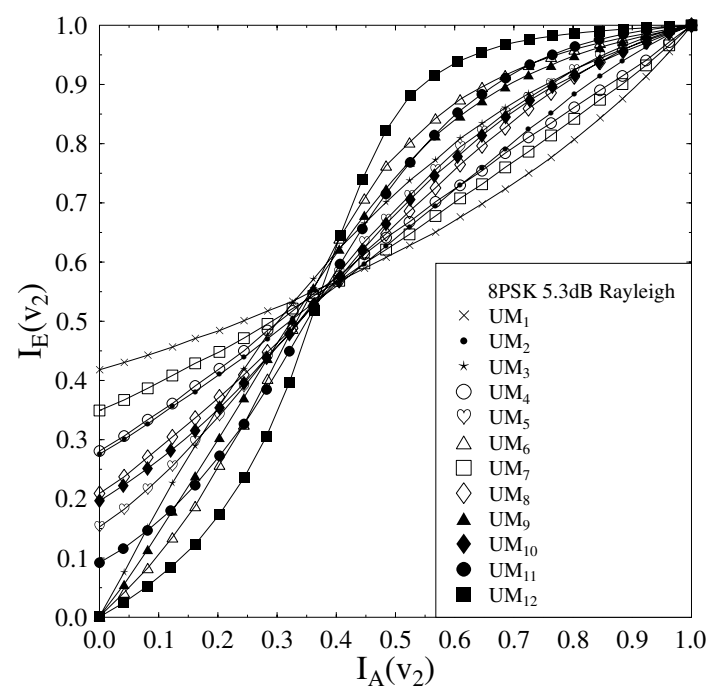

Fig. 4. EXIT functions of the $Q=12$ inner sub-components.

\section{EXIT Chart MATChING}

We adopt the EXIT chart matching algorithm of [12] to jointly match the EXIT functions at both the irregular outer and inner components, as detailed in Section IV-B. The EXIT functions to be considered are described in Equations (4) and (5). More explicitly, we intend to minimise the squared error function defined as follows:

$$
e(i)=\left[f_{v_{2}}\left(i, E_{b} / N_{0}\right)-f_{v_{1}}^{-1}(i)\right] .
$$

Furthermore, the error function should be larger than zero and may be expressed as [12]:

$$
\begin{array}{r}
J\left(\alpha_{1}, \ldots, \alpha_{P}\right)=\int_{0}^{1} e(i)^{2} d i, \quad e(i)>0, \forall i \in[0,1], \quad \text { OR } \\
J\left(\beta_{1}, \ldots, \beta_{Q}\right)=\int_{0}^{1} e(i)^{2} d i, \quad e(i)>0, \forall i \in[0,1],
\end{array}
$$

where $P$ or $Q$ is the number of irregular sub-codes used by either the inner or the outer components, depending on where the matching process is executed. We term the constraint of Equation 13 as condition $C_{1}$. Another constraint we impose here is that of ensuring that Equation (8) is fulfilled and we term this as condition $C_{2}$.

The joint EXIT chart matching algorithm designed for both the inner and outer components in order to find the optimal value of $\alpha_{\text {opt }}$ and $\beta_{\text {opt }}$ can be summarised as follows:

Step 1: Create $P$ outer components (IrCC) and $Q$ inner URC Mapper components (UM).

Step 2: Select one out of the $Q \mathrm{UMs}$, as the inner component to be used.

Step 3: Select a low coding rate for the $\mathrm{IrCC}$, as the initial outer code rate $R_{0}$.

Step 4: Employ the EXIT chart matching algorithm to obtain $\alpha_{\text {opt }}$, subject to the constraints of $C_{1}$ and $C_{2}$.

Step 5: Repeat Step 3 and Step 4 iteratively, until a sufficiently high initial rate $R_{0}$ is obtained.

Step 6: Record the resultant outer EXIT curve. 
Step 7: Execute the matching algorithm of Step 4 for matching $Q$ number of UMs to the resultant outer EXIT curve of Step 6, in order to obtain $\beta_{\text {opt }}$.

Step 8: Record the resultant inner EXIT curve and repeat the EXIT matching process of Step 4, Step 6 and Step 7.

Step 9: Terminate the algorithm, once there is a cross-over between the inner and outer EXIT curves. Store the final values of $\alpha_{\text {opt }}$ and $\beta_{\text {opt }}$.

\section{Simulation Results}

In this section we embark on characterising the proposed Ir-BICMID scheme in terms of its EXIT chart convergence behaviour for transmission over the uncorrelated Rayleigh fading channel. First, we characterise the benchmarker, namely a conventional BICM-ID scheme invoking UP as shown in Figure 5. The conventional BICMID dispenses with the URC, hence the inner component consists of a simple demapper. Therefore in Figure 2, the dashed box representing the BICM-ID scheme is constituted solely by the demapper. The outer code, is constituted by a convolutional code. Figure 5 illustrates the EXIT functions of both the inner and outer components, where the outer code rate was $2 / 3$ associated with $m=3,4$ and 6 number of memory lengths.

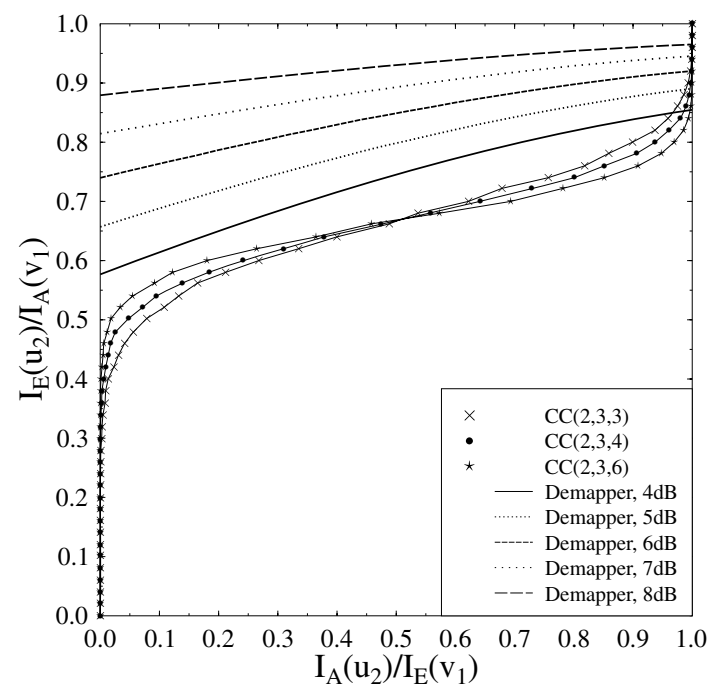

Fig. 5. EXIT functions of the BICM-ID inner and outer components, transmitting over an uncorrelated Rayleigh fading channel.

Observe from Figure 5 that the inner demapper does not reach the point of convergence at $(1,1)$. Furthermore, there is a mismatch between the corresponding EXIT curve shapes, indicating an $E_{b} / N_{0}$ 'loss' with respect to the capacity. A further $E_{b} / N_{0}$ improvement is achieved upon introducing an IrCC outer code, which reduces the area of the open EXIT tunnel, as shown in Figure 6, but still exhibits a 'larger-than-necessary' EXIT tunnel. Figure 6 shows that the shape of the outer IrCC EXIT function is better matched to that of the inner codes, as indicated by the dotted line shifting upwards, when the channel's $E_{b} / N_{0}$ value increases.

Let us finally employ the EXIT matching algorithm described in Section V, invoking the IrCC, IrURC as well as Ir-Mapper schemes. The shapes of the EXIT functions enables us to reduce the open EXIT tunnel area and hence to create a near-capacity Ir-BICM-ID scheme. As a further benefit, we are able to shift the inner EXIT function closer to the $(1,1)$ point for the sake of achieving an infinitesimally low BER.

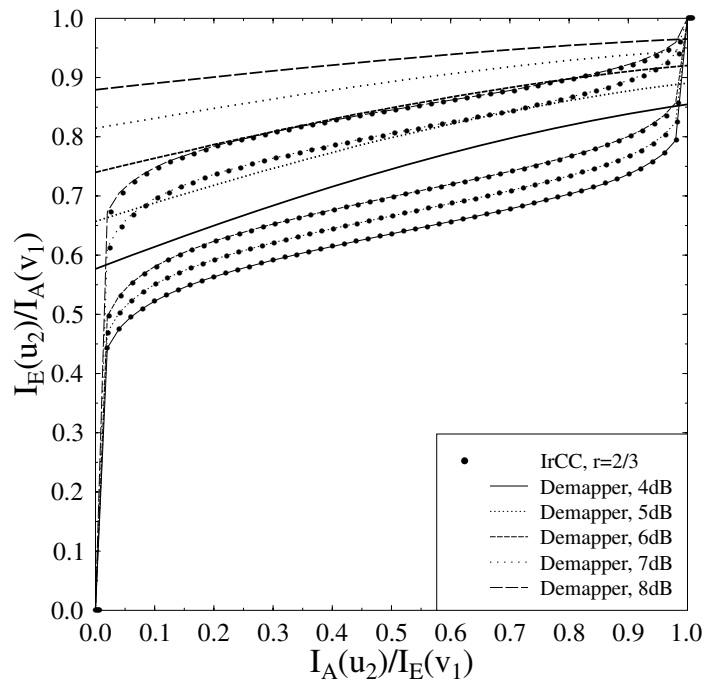

Fig. 6. EXIT functions of the BICM-ID inner and outer components, using an $r=2 / 3 \mathrm{IrCC}$ as the outer component, when communicating over an uncorrelated Rayleigh fading channel.

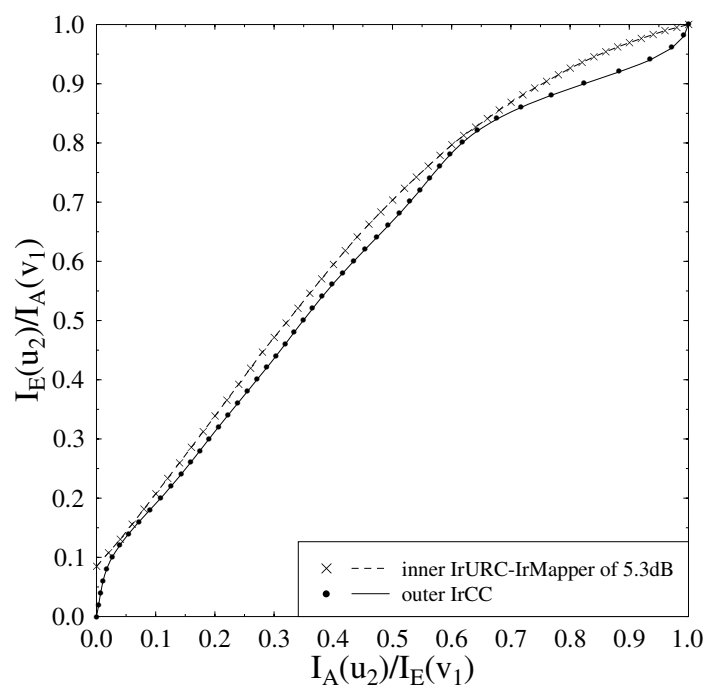

Fig. 7. EXIT functions of the Ir-BICM-ID schemes designed for transmission over an uncorrelated Rayleigh fading channel at $E_{b} / N_{0}=5.3 \mathrm{~dB}$.

We observe from Figure 7 that the open EXIT tunnel of the resultant scheme is narrow and reaches the point of convergence at $(1,1)$. However, since the number of iterations required increases, the decoding complexity is also increased. Note that the proposed IrBICM-ID scheme has advantages over the bit-interleaved irregular modulation scheme of [6], when we further explore the effects of various mapping schemes combined with URCs having different memory lengths. This gives us the flexibility of adjusting the EXIT curve shape in order to achieve a low BER, without having to change the number of modulated constellation points, which would require complex, state-of-the-art AMC. Furthermore, we employ the joint EXIT matching algorithm to produce flexible inner and outer component codes. The complexity imposed by the iterations between the IrURC and IrMapper schemes is low compared to that of the outer IrCC.

The EXIT function of Figure 7 was recorded for the EXIT chart matching algorithm of Section V. The resultant weighting coefficients 
of $\alpha_{\text {opt }}$ and $\beta_{\text {opt }}$ are as follows:

$$
\begin{aligned}
\alpha_{\text {opt }}= & {\left[\alpha_{1}, \ldots, \alpha_{17}\right] } \\
= & {[0.0226995,0.0530819,0.0717173,0,0.0299283,} \\
& 0.0466581,0.0952706,0.023804,0.00206623, \\
& 0.0331461,0.0160705,0.19097,0,0,0,0.0967027, \\
& 0.317832], \\
\beta_{\text {opt }}= & {\left[\beta_{1}, \ldots, \beta_{12}\right] } \\
= & {[0,0,0.340268,0,0.550512,0,} \\
& 0,0,0.109219,0,0,0] .
\end{aligned}
$$

The theoretical Discrete-input Continuous-output Memoryless Channel's (DCMC) capacity is plotted in comparison to the maximum achievable capacity of the proposed Ir-BICM-ID scheme in Figure 8. Note that the achieveable capacity of the Ir-BICM-ID scheme is close to the DCMC's capacity. For example, at SNR $=6 \mathrm{~dB}$ the capacity gap between the theoretical value and the proposed coded modulation scheme is only $0.28 \mathrm{~dB}$. This confirms the benefits of the proposed EXIT chart matching approach.

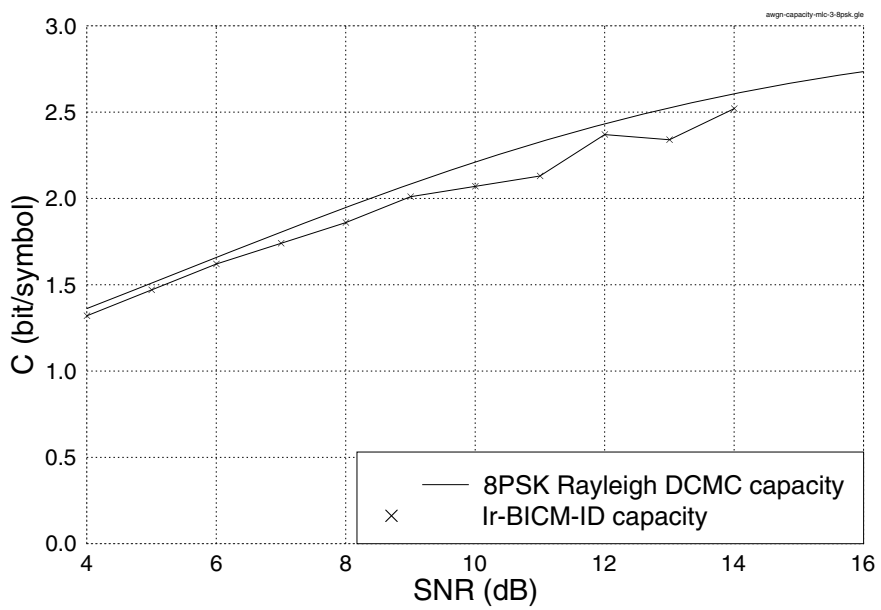

Fig. 8. The maximum effective throughput of the proposed Ir-BICM-ID scheme in comparison to the theoretical DCMC capacity plot [18]. The transmission is over 8PSK uncorrelated Rayleigh fading channel.

\section{CONCLUSIONS}

In conclusion, a novel Ir-BICM-ID scheme was proposed in this paper. This scheme invokes a combined IrCC, IrURC and IrMapper arrangement. Various EXIT curves were produced for both inner and outer component codes. A useful joint inner-outer EXIT matching algorithm was employed for obtaining a narrow but still open EXIT tunnel, which indicated near-capacity operation. Our results demonstrate that in contrast to the classic BICM-ID schemes, the proposed arrangement is capable of attaining an infinitesimally low BER. Furthermore, as shown in Figure 8, the system is capable of operating within about $0.3 \mathrm{~dB}$ from the DCMC's capacity in the low$\mathrm{SNR}$ region, say for $\mathrm{SNR} \leq 6 \mathrm{~dB}$.

\section{REFERENCES}

[1] E. Zehavi, "8-PSK Trellis Codes for a Rayleigh Fading Channel," IEEE Transactions on Communications, vol. 40, pp. 873-883, May 1992.

[2] X. Li and J. A. Ritcey, "Bit-interleaved Coded Modulation with Iterative Decoding," IEEE Communications Letters, vol. 1, pp. 169-171, November 1997.

[3] S. T. Brink, "Convergence Behavior of Iteratively Decoded Parallel Concatenated Codes," IEEE Transaction on Communication, vol. 49, no. 10 , pp. $1727-1737$.
[4] N. H. Tran and H. H. Nguyen, "Signal Mappings of 8-Ary Constellations for BICM-ID Systems over a Rayleigh Fading Channel," IEICE Transcations Letter on Communication, pp. 4083-4086, October 2005.

[5] F. Simoens, H. Wymeersch and M. Moeneclaey, "Multi-dimensional Mapping for Bit-Interleaved Coded Modulation," Vehicular Technology Conference, Spring, vol. 2, pp. 733-737, June 2005.

[6] F. Schreckenbach and G. Bauch, "Bit-Interleaved Coded Irregular Modulation," European Transactions on Telecommunications, vol. 7, pp. 269282, April 2006.

[7] L. Hanzo, C. H. Wong, and M. S. Yee, Adaptive Wireless Transceivers: TurboCoded, TurboEqualized and SpaceTime Coded TDMA, CDMA, and OFDM Systems. Chichester, UK: John Wiley IEEE Press, August 2002.

[8] K. R. Narayanan, "Effect of Precoding on the Convergence of Turbo Equalization for Partial Response Channels," IEEE Journal on Selected Areas in Communications, vol. 19, pp. 686-698, Apr. 2001.

[9] F. Simoens, H. Wymeersch and M. Moeneclaey, "Design and Analysis of Linear Precoders for Bit-Interleaved Coded Modulation with Iterative Decoding," in International Symposium on Turbo Codes and Related Topics, (Munich, Germany), April 2006.

[10] L. Szczecinski, H. Chafnaji and C. Hermosilla, "Modulation Doping for Iterative Demapping of Bit-Interleaved Coded Modulation," IEEE Communications Letters, vol. 9, pp. 1031-1033, 2005.

[11] M. Tüchler, "Design of Serially Concatenated Systems Depending on The Block Length," IEEE Transactions On Communications, vol. 52, pp. 209-218, February 2004.

[12] M. Tüchler and J. Hagenauer, "EXIT Charts of Irregular Codes," IEEE Conference on Information Sciences and Systems, pp. 748-753, March 2002.

[13] M. Tüchler, "Convergence prediction for iterative decoding of threefold concatenated systems," IEEE Global Telecommunications Conference, vol. 2, pp. 1358-1362, Nov. 2002.

[14] A. Ashikhmin, G. Kramer and S. T. Brink, "Extrinsic Information Transfer Functions: Model and Erasure Channel Properties," IEEE Transactions on Information Theory, vol. 50, pp. 2657-2673, November 2004.

[15] R. G. Maunder, J. Wang, S. X. Ng, L-L. Yang and L. Hanzo, "On the Performance and Complexity of Irregular Variable Length Codes for Near-Capacity Joint Source and Channel Coding," IEEE Transactions on Wireless Communications (in press), 2007. http://eprints.ecs.soton.ac.uk/14467/.

[16] G. Ungerböck, "Channel Coding with Multilevel/Phase Signals," IEEE Transactions on Information Theory, vol. 28, pp. 55-67, January 1982.

[17] U. Wachsmann, R. F. H. Fischer and J. B. Huber, "Multilevel Codes: Theoretical Concepts and Practical Design Rules," IEEE Transaction on Information Theory, vol. 45, pp. 1361-1391, July 1999.

[18] L. Hanzo, S. X. Ng, T. Keller and W. Webb, Quadrature Amplitude Modulation. Chichester, UK : John Wiley and Sons, 2004. 